\title{
CALET Ultra Heavy Cosmic Ray Observations on the ISS
}

\author{
Brian Flint Rauch* \\ Department of Physics and McDonnell Center for the Space Sciences, Washington University, \\ St. Louis, MO 63130 USA \\ E-mail: brauch@physics.wustl.edu

\section{Walter Robert Binns} \\ Department of Physics and McDonnell Center for the Space Sciences, Washington University, \\ St. Louis, MO 63130 USA \\ E-mail: wrb@wust 1.edu
}

\section{for the CALET Collaboration}

The CALorimetric Electron Telescope (CALET) on the International Space Station (ISS) has made cosmic-ray observations since shortly after its launch in August 2015. The heart of CALET, its main calorimeter (CAL), continues to make the direct measurements of the highest energy cosmic-ray total electron spectrum it was designed for. The CAL has also observed gamma rays and cosmic-ray (CR) nuclei, and it has measured energy spectra as well as secondary to primary ratios of the more abundant $\mathrm{CR}$ nuclei through ${ }_{26} \mathrm{Fe}$. In addition, the CAL has measured the ultra-heavy cosmic ray (UHCR) nuclei heavier than ${ }_{26} \mathrm{Fe}$, and it has demonstrated the dynamic range to measure $\mathrm{CR}$ nuclei from ${ }_{1} \mathrm{H}$ to ${ }_{40} \mathrm{Zr}$. Using the main high energy (HE) CAL trigger mode particle energies can be reconstructed from the energy deposition in the total absorption calorimeter (TASC), and there is also a high duty cycle ( $90 \%)$ UHCR trigger providing an expanded geometric acceptance that is $\sim 6 \times$ that for events fully contained by the CAL. In $\sim 5$ years the UHCR trigger will collect a data set comparable to that so far collected by the balloonborne SuperTIGER instrument. Preliminary CALET results presented at the last ICRC were in reasonable agreement with SuperTIGER relative abundances of even charge UHCR nuclei in a similar energy range. Both of these measurements are complemented by the $\sim 1 / 3$ smaller lowerenergy space-based ACE-CRIS measurements. We present the current status and future plans for the CALET UHCR analysis.

36th International Cosmic Ray Conference -ICRC2019-

July 24th - August 1st, 2019

Madison, WI, U.S.A.

\footnotetext{
* Speaker.

${ }^{\dagger}$ CALET supported by JAXA in Japan, ASI in Italy and in the USA by NASA grant \#NNX11AE02G.

${ }^{\ddagger}$ for collaboration list see PoS(ICRC2019)1177
} 


\section{Introduction}

The CALorimetric Electron Telescope (CALET) on the International Space Station (ISS) is a Japanese-Italian-US astroparticle observatory that was launched on August 19, 2015 and has been collecting scientific data since October 13, 2015 [1]. As its name suggests, the principal science objective of CALET is to directly measure the total cosmic-ray electron flux $\left(\mathrm{e}^{-}+\mathrm{e}^{+}\right)$to the highest energies ( $1 \mathrm{GeV}$ to $20 \mathrm{TeV})[2,3,4]$ with the main calorimeter (CAL), shown in the CALET instrument package in the left plot of Fig. 1. The CAL is also cable of measuring gamma rays $(10 \mathrm{GeV}$ to $10 \mathrm{TeV}$ ) [5, 6] and cosmic-ray nuclei (up to $1,000 \mathrm{TeV}$ ) $[7,8,9,10]$. In addition to the CAL, left plot of Fig. 1 also shows the CALET Gamma-ray Burst Monitor (CGBM) [11], which can make simultaneous observations with the CAL.
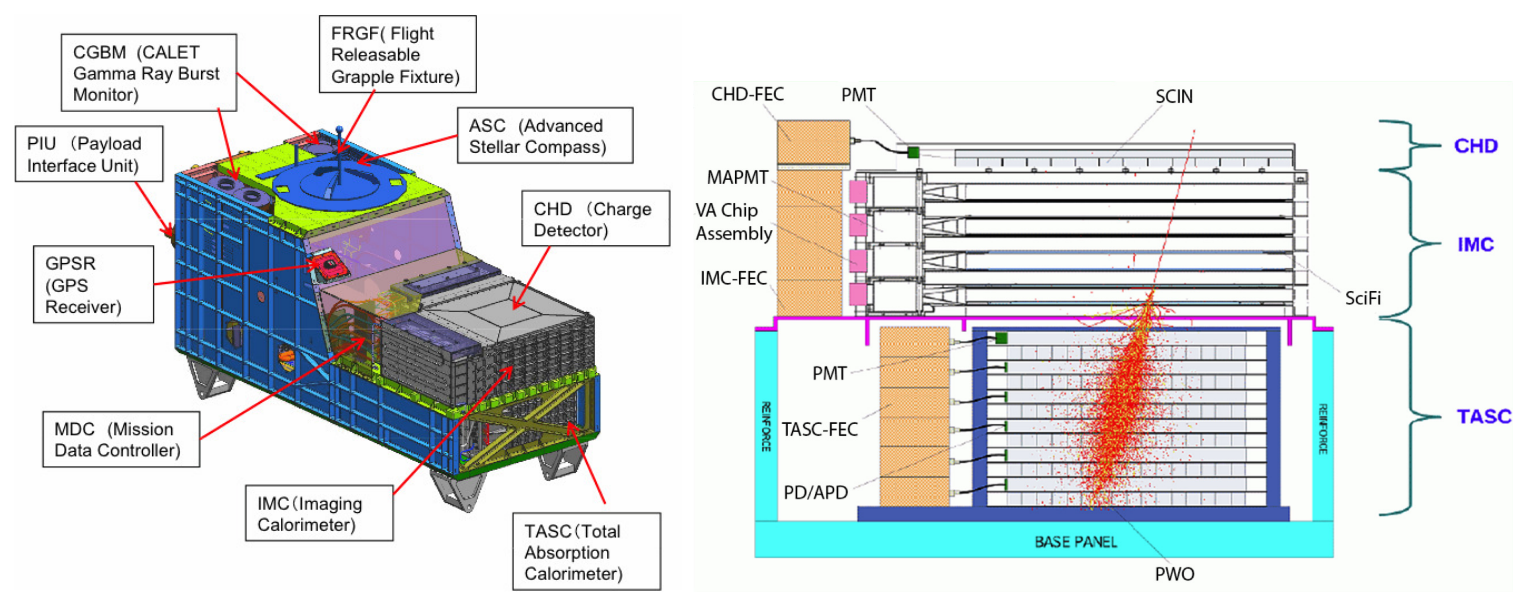

Figure 1: Left: CALET instrument package showing CAL and CGBM subsystems. Right: CAL side-view showing CHD, IMC, and TASC detectors with an example image of a simulated $1 \mathrm{TeV}$ electron shower.

The CAL is comprised of three detector systems, shown in more detail in a side view in the right plot of Fig. 1. At the top is the charge detector (CHD), comprised of $\mathrm{x}$ and y layers of 14 scintillator paddles, each $32 \mathrm{~mm}$ wide by $10 \mathrm{~mm}$ thick by $450 \mathrm{~mm}$ long. Below that is the imaging calorimeter (IMC), which is $156.5 \mathrm{~mm}$ tall and made of 8 layers of $\mathrm{x}$ and y scintillating fibers (1 $\mathrm{mm}$ square and $448 \mathrm{~mm}$ long) interleaved with 3.0 radiation lengths $\left(X_{\circ}\right)$ of tungsten (5 layers 0.2 $X_{\circ}$ thick above 2 layers $1.0 X_{\circ}$ ). At the bottom is the total absorption calorimeter (TASC), made of $6 \mathrm{x}$ and y layers of 16 lead tungstate (PWO) scintillator logs (19 $\mathrm{mm}$ wide by $20 \mathrm{~mm}$ tall by $326 \mathrm{~mm}$ long) having a depth of $27 X_{\circ}$. The CHD, along with the IMC in some cases, provides the primary particle charge identification. The segmented nature of all three detectors is utilized in track reconstruction, which relies primarily on the IMC. Particle energy is determined from the calorimeters, relying mostly on the TASC.

\section{Ultra-Heavy Cosmic Rays}

The ultra-heavy cosmic rays (UHCR), ${ }_{30} \mathrm{Zn}$ and higher charge elements, provide insight into the origins of the galactic cosmic rays. Fig. 2 shows the relative abundances of elements $(1 \leq Z \leq$ 40) for cosmic rays with energies of $2 \mathrm{GeV}$ /nucleon compared with Solar System (SS) abundances 
normalized to ${ }_{14} \mathrm{Si}$. These two samples of galactic matter are generally consistent, with most of the differences accounted for by cosmic ray spallation while traveling from their source and by acceleration efficiencies. In the cosmic rays we see that ${ }_{26} \mathrm{Fe}$ is $\sim 5 \times 10^{3}$ times less abundant than ${ }_{1} \mathrm{H}$, and that the UHCR with charges $30 \leq Z \leq 40$ are $\sim 10^{5}$ times less abundant than ${ }_{26} \mathrm{Fe}$. Single-element resolution UHCR measurements have so far only been made up to ${ }_{40} \mathrm{Zr}$ by the TIGER [12] and SuperTIGER [13] balloon-borne instruments at GeV/nuc energies, and by the ACE-CRIS [14] space based instrument at hundreds of MeV/nuc. The UHCR composition shows enhancement from material produced in massive stars, both in stellar outflows during the stars' lives and in supernova ejecta. This suggests that a significant fraction of the cosmic rays originate in $\mathrm{OB}$ associations, which is where the majority of supernova that are believed to accelerate the galactic cosmic rays occur. The fact that the cosmic-ray source appears to be enhanced in massive star material compared to SS suggests that UHCR observations could help constrain the relative contributions of supernovas and binary neutron star mergers to the heavy r-process elements.

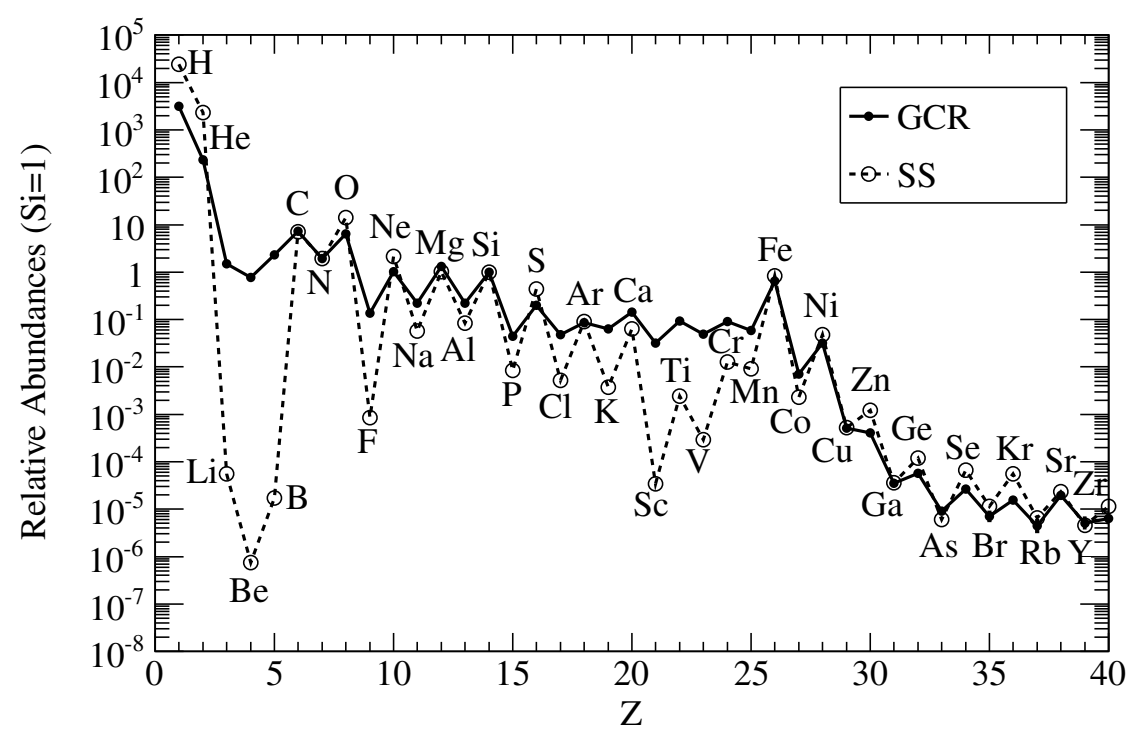

Figure 2: Solar System (SS) [15] and Galactic cosmic-ray (GCR) relative abundances at $2 \mathrm{GeV} /$ nuc $(1 \leq$ $Z \leq 2$ from [16], $Z=3$ from [17], $4 \leq Z \leq 28$ from [18], $Z=29$ from [12], and $28 \leq Z \leq 40$ from [13].) normalized to ${ }_{14} \mathrm{Si}=1$.

CALET observations will contribute significantly to the statistics of the UHCR data set and complement existing measurements. The ultra-heavy (UH) event trigger on CALET, which requires events only pass through the CHD and top half of the IMC, will collect in $\sim 5$ years UHCR statistics similar to those achieved in the first flight of the balloon-borne SuperTIGER instrument [19]. CALET observes a similar energy range to TIGER/SuperTIGER, but requires corrections for different systematic effects. The balloon observations must be corrected for energy losses and nuclear interactions occurring in the atmosphere, while the ISS based measurements in a $51.6^{\circ}$ inclination orbit are subject to screening by the geomagnetic field. The ACE-CRIS UHCR observations occur in space outside of the geomagnetic field in a complementary lower energy range. Finally, CALET has the unique capability to measure cosmic-ray abundances in the $1 \leq Z \leq 40$ 
charge range with a single instrument, which provides a check of the cross calibrations used in other measurements.

\section{Analysis}

\subsection{Previous Results}

Preliminary CALET UHCR results reported at the last ICRC were consistent with those published for SuperTIGER[20]. This analysis was based on $\sim 13$ months of data passing the CALET ultra-heavy (UH) trigger, which provides an increased acceptance by requiring events only pass through the CHD and the top four layers of the IMC to give a geometry factor of $0.44 \mathrm{~m}^{2}$ sr. Figure 3 shows the charge histogram for this analysis on the left plot, and on the right is a plot comparing CALET and SuperTIGER even- $Z$ elemental abundances. The data shown are for UH trigger events that had reconstructed trajectories, were within $60^{\circ}$ of vertical, passed CHD charge consistency cuts, and which had a geomagnetic vertical cutoff rigidity $>4 \mathrm{GV}$. The rigidity cut is used in UH trigger event analysis to compensate for the lack of information on event energy from the TASC to screen out lower energy particles below minimum ionization that would confound the charge determination. The abundances were determined by a multiple-Gaussian fit with integer charge means and fixed $\sigma=0.35$.
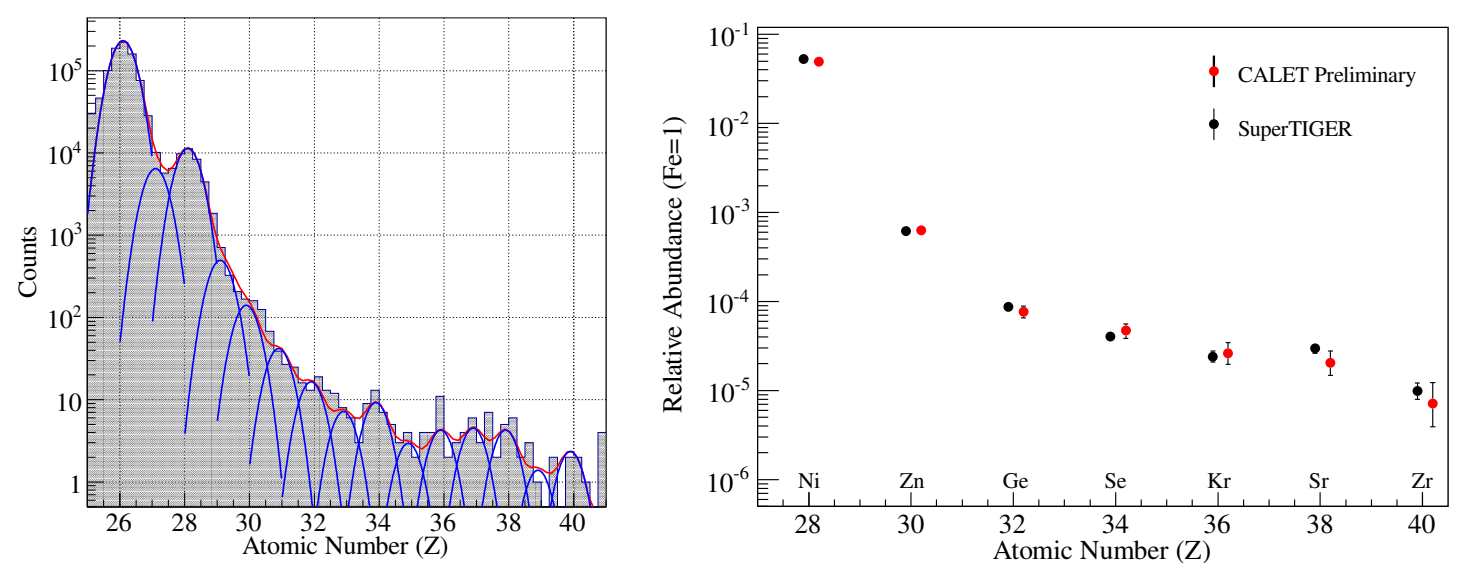

Figure 3: Left: Preliminary CALET UHCR charge histogram with 4 bins/charge unit for UH trigger events within $60^{\circ}$ of vertical and for a geomagnetic vertical cutoff rigidity $>4 \mathrm{GV}$. Right: Even- $Z$ relative abundances $\left({ }_{26} \mathrm{Fe}=1\right.$ ) for CALET (red points) compared with SuperTIGER (black points) [13].

\subsection{Current Status}

Analysis since the last ICRC has focused on increasing the CALET UHCR charge resolution through improved data corrections and selections. The primary time and position response corrections for CALET are based on ${ }_{2} \mathrm{He}$ and ${ }_{1} \mathrm{H}$, and to improve resolution in UH-range we have supplemented these corrections in the CHD with ones using ${ }_{26} \mathrm{Fe}$ nuclei. For each CHD paddle the ${ }_{26} \mathrm{Fe}$ peak is fit in time intervals where there are a minimum of $\sim 500$ events in each peak. For each time step the paddle signals are multiplied by the ratio of the overall ${ }_{26} \mathrm{Fe}$ peak mean to the one for 
the paddle's mean. The left plot of Fig. 4 shows the ${ }_{26} \mathrm{Fe}$ peak mean time dependence for a sample of CHDY paddles, and the histogram on the right shows the ${ }_{26} \mathrm{Fe}$ means for all paddles before and after the UH-analysis time correction is applied.
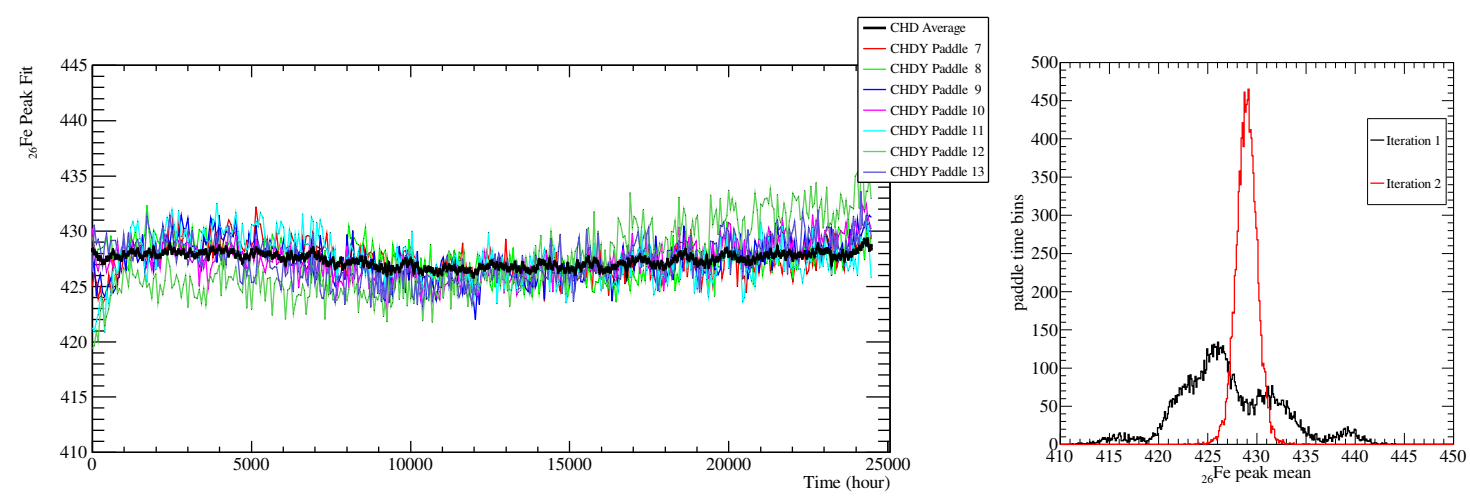

Figure 4: Left: Time dependence of ${ }_{26} \mathrm{Fe}$ means in half of the CHDY paddles before UH-analysis corrections. Right: Histogram showing all CHD paddle ${ }_{26} \mathrm{Fe}$ means before (Iteration 1, black) and after (Iteration 2 , red) UH time corrections are applied.

The UH-analysis position-dependent corrections similarly rely on both ${ }_{14} \mathrm{Si}$ and ${ }_{26} \mathrm{Fe}$ mean fits. Each CHD paddle is divided into 42 segments with $1 / 3$ the width of a paddle. For each segment ${ }_{14} \mathrm{Si}$ and ${ }_{26} \mathrm{Fe}$ peaks are fit for the histograms of $\mathrm{UH}$-analysis data with the time corrections applied. Initially, the position corrections were applied with ${ }_{26} \mathrm{Fe}$ mean ratios as with the time corrections, but now a linear correction is applied using both ${ }_{14} \mathrm{Si}$ and ${ }_{26} \mathrm{Fe}$ segment means. The left plot of Fig. 5 shows the CHDX segment ${ }_{26} \mathrm{Fe}$ means before (Iteration 1, black) and after (Iteration 2, red) the UH-analysis position-dependent correction is applied. The middle plot in Fig. 5 shows CHDX segment ${ }_{14} \mathrm{Si}$ peak means before and after the ${ }_{26} \mathrm{Fe}$ ratio position correction is applied, and the right figure shows the same ${ }_{14} \mathrm{Si}$ means when the ${ }_{14} \mathrm{Si}$ and ${ }_{26} \mathrm{Fe}$ based correction is applied. The linear correction was used so that all of the paddles will have more uniform gain responses, which will improve charge consistency selections and charge assignments.
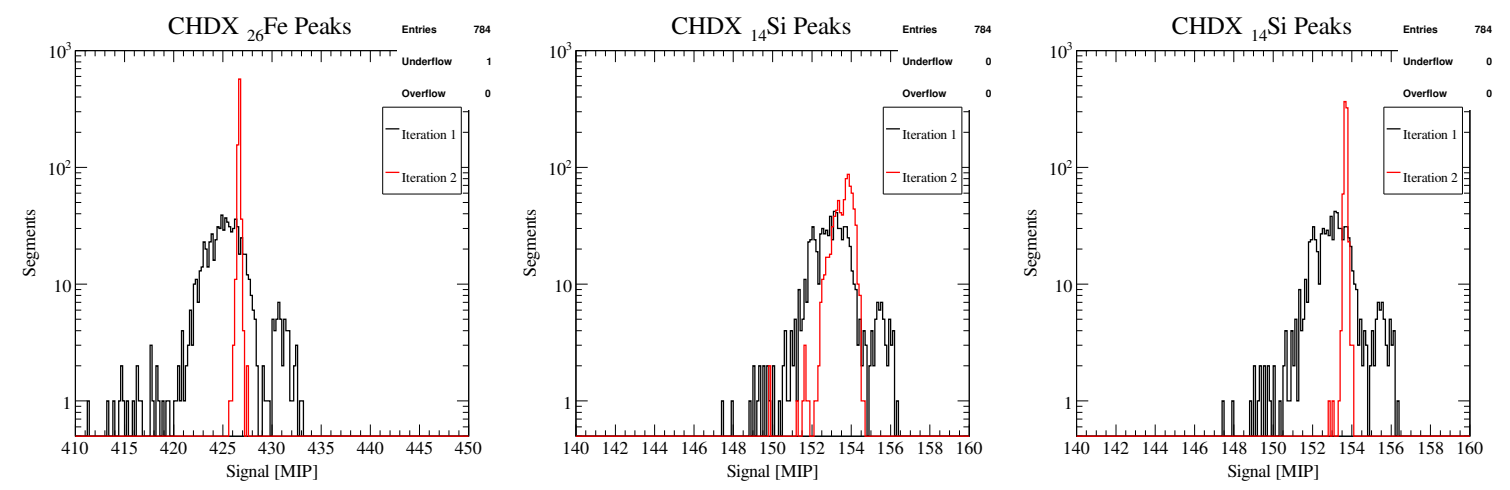

Figure 5: Left: CHDX segment ${ }_{26} \mathrm{Fe}$ peak means before (Iteration 1, black) and after (Iteration 2, red) ${ }_{14} \mathrm{Si}$ and ${ }_{26} \mathrm{Fe}$ dependent position corrections are applied. Middle: Histogram of CHDX segment ${ }_{14} \mathrm{Si}$ peak means before and after ${ }_{26} \mathrm{Fe}$ dependent position corrections are applied. Right: Histogram of CHDX segment ${ }_{14} \mathrm{Si}$ peak means before and after ${ }_{14} \mathrm{Si}$ and ${ }_{26} \mathrm{Fe}$ dependent position corrections are applied. 
After time- and position-dependent corrections are applied, selection cuts are made to optimize the UHCR resolution. These are quite similar to those used in the previous ICRC analysis, by also requiring trajectory reconstruction and having the same $>4 \mathrm{GV}$ vertical cutoff rigidity threshold requirement. In the present analysis two charge consistency cuts were employed, with Fig. 6 left showing that for CHDX and CHDY charges derived from the total layer signals and the middle plot showing that for charges derived from the highest paddle signals in each layer. The charges used in the former selection include more signal from backscatter, while the latter is more focused on the signal from the primary particle track. Another selection that screens out events with high signal contributions outside of the primary particle track, shown in the right plot of Fig. 6 for CHDX, sets a limit on the relative strength of the third highest paddle signal in each CHD layer.
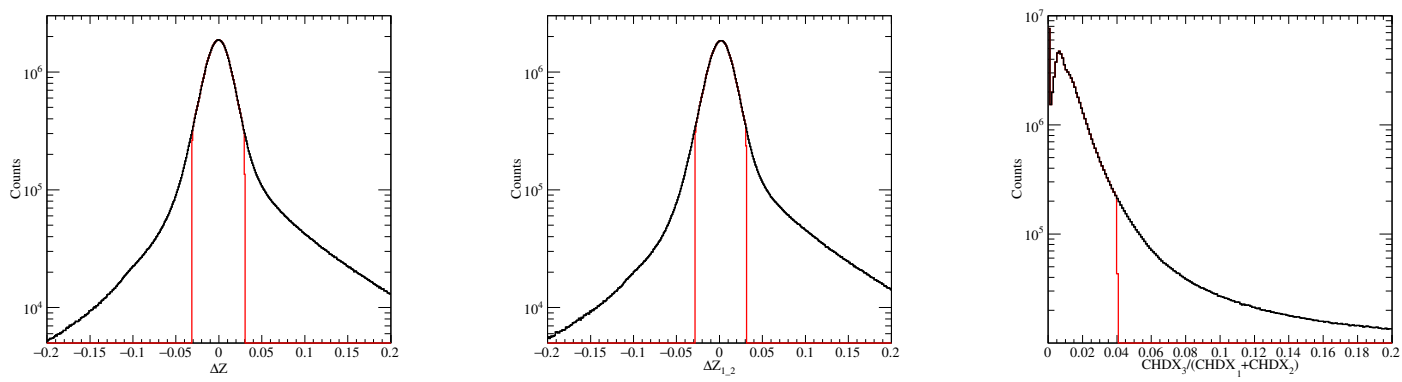

Figure 6: Left: $C H D$ layer $2 \sigma$ total signal charge consistency cut on $\Delta Z=\left(Z_{C H D X}-Z_{C H D Y}\right) /\left(Z_{C H D X}+\right.$ $\left.Z_{C H D Y}\right)$. Middle: CHD highest two paddles $2 \sigma$ charge consistency cut on $\Delta Z_{12}$. Right: CHDX selection limiting relative strength of third highest paddle.

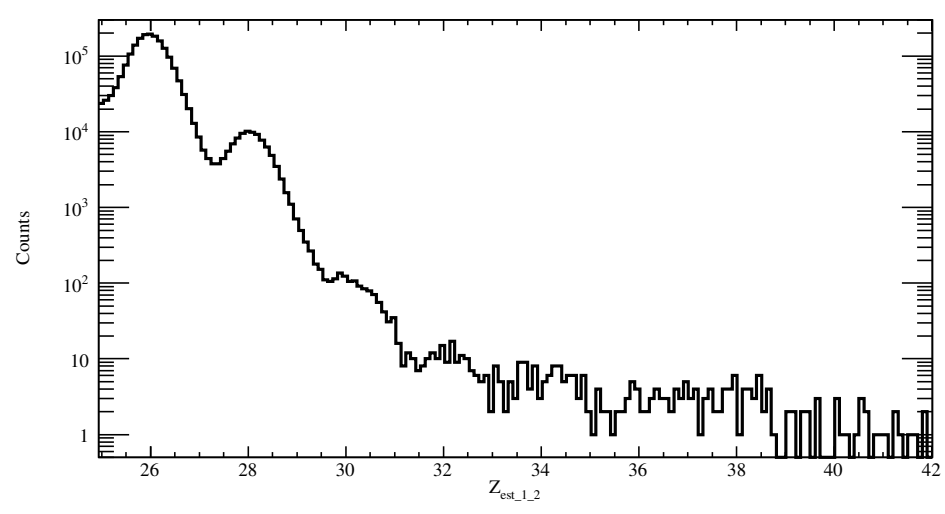

Figure 7: CALET UHCR charge histogram for present analysis with 10 bins/charge unit for UH trigger events within $45^{\circ}$ of vertical and for a geomagnetic vertical cutoff rigidity $>4 \mathrm{GV}$.

The present analysis also uses $\mathrm{a} \leq 45^{\circ}$ incidence angle cut selection, which is more restrictive than the $\leq 60^{\circ}$ cut used in the previous ICRC analysis. One final selection applied is a minimum threshold for the average signal from the top four layers of the IMC, which cuts most of the nuclei below ${ }_{26} \mathrm{Fe}$ and helps reduce the low-energy tail in the UH-range. Figure 7 shows the CALET charge histogram for the present analysis on $\sim 34$ months of data. This histogram has 10 bins per charge unit, which is finer than the 4 bins per charge unit in the 2017 ICRC histogram. The peaks 
are better defined, showing that improved charge resolution can be achieved with stronger selection cuts. Spillover from the tails of lower- $Z$ high statistics peaks is mostly confined to below ${ }_{32} \mathrm{Ge}$.

\subsection{Ongoing and Future Work}

Progress continues in a number of areas for which results are not ready for this ICRC. The charge assignments shown in this analysis are fairly simplistic, $\propto Z^{1 / 1.8}$, but a charge model accounting for scintillator saturation following the method in [21], fit to incidence angle and relative CHD signal bins, is in development. A model for the geomagnetic field has been implemented that provides the field strength as a function of latitude, longitude, altitude and East-West angle. Work continues on using this model to provide trajectory-dependent rigidity thresholds for the CALET events, which will allow a more refined selection than the vertical cutoff rigidity one currently in use. Also along these lines, we have started development on a model that will trace each event trajectory back through a full geomagnetic field model to more precisely determine its geomagnetic threshold.

\section{Conclusions}

Preliminary CALET UHCR abundances are consistent with previous measurements, and work continues toward final results. CALET continues to return excellent data from the ISS, and it is expected to continue operations for several more years. This will allow CALET to contribute significantly to the total UHCR data set, as well as complement the measurements of other balloonand space-borne instruments.

\section{References}

[1] Y. Asaoka for the CALET Collaboration, The CALorimetric Electron Telescope (CALET) on the International Space Station, in proceedings of The 36th International Cosmic Ray Conference, PoS (ICRC2019) 678 (2019).

[2] S. Torii for the CALET Collaboration, Extended Measurement of Cosmic-Ray Electron and Positron Spectrum from CALET on the ISS, in proceedings of The 35th International Cosmic Ray Conference, POS (ICRC2019) 182 (2019).

[3] H. Motz for the CALET Collaboration, Analysis of CALET Data for Anisotropy in Electron+Positron Cosmic Rays, in proceedings of The 36th International Cosmic Ray Conference, PoS ( ICRC2019) 386 (2019).

[4] S. Miyake, Y. Migita, Y.Asaoka et al. for the CALET Collaboration, Solar Modulation of Galactic Cosmic-Ray Electrons Measured with CALET, in proceedings of The 36th International Cosmic Ray Conference, POS (ICRC2019) 674 (2019).

[5] N. Cannady for the CALET Collaboration, CALET Upper Limits on GeV-energy Gamma-Ray Burst Emission, in proceedings of The 36th International Cosmic Ray Conference, POS ( ICRC2019) 858 (2019).

[6] M. Mori and Y. Asaoka for the CALET Collaboration, High-Energy Gamma-ray Observations Using the CALorimetric Electron Telescope (CALET) on the ISS, in proceedings of The 36th International Cosmic Ray Conference, PoS (ICRC2019) 291 (2019). 
[7] P.S. Marrocchesi for the CALET Collaboration, Measurement of the Proton Spectrum with CALET on the ISS, in proceedings of The 36th International Cosmic Ray Conference, POS ( ICRC2019) 531 (2019).

[8] Y. Akaike for the CALET Collaboration, Measurements of Heavy Cosmic Ray Nuclei Fluxes with CALET, in proceedings of The 36th International Cosmic Ray Conference, POS ( ICRC2019) 386 (2019).

[9] P. Maestro for the CALET Collaboration, Measurement of the energy spectra of carbon and oxygen nuclei in cosmic rays with CALET in proceedings of The 36th International Cosmic Ray Conference, POS (ICRC2019) 582 (2019).

[10] A. Bruno, G. de Nolfo and A. Ficklin for the CALET Collaboration, Space Weather Observations during September 2017 with CALET on the International Space Station in proceedings of The 36th International Cosmic Ray Conference, POS ( ICRC2019) 11 (2019).

[11] Y. Kawakubo for the CALET Collaboration, Gamma-ray burst observations with the CALET Gamma-ray Burst Monitor, in proceedings of The 36th International Cosmic Ray Conference, PoS (ICRC2019) 738 (2019).

[12] B.F. Rauch et al., Cosmic Ray origin in OB Associations and Preferential Acceleration of Refractory Elements: Evidence from Abundances of Elements ${ }_{26}$ Fe through ${ }_{34} \mathrm{Se}, \mathrm{ApJ}, 697$ (2009) 2083-2088, [arXiv:0906.2021].

[13] R.P. Murphy et al., Galactic Cosmic Ray Origins and OB Associations: Evidence from SuperTIGER Observations of Elements ${ }_{26} \mathrm{Fe}$ Through ${ }_{40} \mathrm{Zr}, \mathrm{ApJ}, \mathbf{8 3 1}$ (2016) 2083-2088, [arXiv: 1608.08183 ].

[14] W.R. Binns, M.H. Israel, M.E. Wiedenbeck, et al., Elemental Source Composition Measurements and the Origin of Galactic Cosmic Rays âĂS ACE-CRIS Observations of UH Elements, in proceedings of The 36th International Cosmic Ray Conference, PoS (ICRC2019) 673 (2019).

[15] K. Lodders, Solar System Abundances and Condensation Temperatures of the Elements, ApJ, 591 (2003) 1220-1247.

[16] T. Sanuki et al., Precise Measurement of Cosmic-Ray Proton and Helium Spectra with the BESS Spectrometer, ApJ, 545 (2000) 148-155, [arXiv: astro-ph/ 0002481 ].

[17] M. Aguilar et al., Isotopic Composition of Light Nuclei in Cosmic Rays: Results from AMS-01, ApJ, 736 (2011) 105-116, [arXiv:1106.2269].

[18] J.J. Engelmann et al., Charge composition and energy spectra of cosmic-ray nuclei for elements from Be to NI - Results from HEAO-3-C2, A\&A, 233 (1990) 96-111.

[19] B.F. Rauch for the CALET Collaboration, Predicted CALET Measurements of Heavy and Ultra-Heavy Cosmic Ray Nuclei, in proceedings of The 34th International Cosmic Ray Conference, POS (ICRC2015) 627 (2015).

[20] B.F. Rauch and Y. Akaike for the CALET Collaboration, Status of the CALET Ultra Heavy Cosmic Ray Analysis in proceedings of The 35th International Cosmic Ray Conference, PoS ( ICRC2017) (2017).

[21] P.S. Marrocchesi et al., Beam test performance of a scintillator-based detector for the charge identification of relativistic ions, NIMPA, 659 (2011) 477-483. 